\title{
L'inéluctable incomplétude des politiques industrielles régionales et européennes
}

Les leçons de la reconversion des industries de la Défense dans les années 1990

Bernard Jullien et Vincent Frigant

\section{(2) OpenEdition Journals \\ Édition électronique \\ URL : http://journals.openedition.org/ei/262 \\ DOI : $10.4000 /$ ei.262 \\ ISSN : 2553-1891 \\ Éditeur \\ Association Économie et Institutions}

Édition imprimée

Date de publication : 30 octobre 2009

Pagination : 139-167

ISSN : $1775-2329$

Référence électronique

Bernard Jullien et Vincent Frigant, «L'inéluctable incomplétude des politiques industrielles régionales et européennes », Économie et institutions [En ligne], 12-13 | 2009, mis en ligne le 31 janvier 2013, consulté le 20 avril 2019. URL : http://journals.openedition.org/ei/262 ; DOI : 10.4000/ei.262 


\title{
L'inéluctable incomplétude des politiques \\ industrielles régionales et européennes : les leçons de la reconversion des industries de la défense dans les années 1990
}

\author{
Bernard Jullien ${ }^{1}$ et Vincent Frigant ${ }^{2}$
}

\section{Introduction}

Les industries de défense étaient traditionnellement présentées comme étant doublement atypiques ou exotiques.

- On les disait ancrées dans des logiques technoproductives très spécifiques et de ce fait difficilement comparables à d'autres industries.

- On soulignait qu'elles étaient historiquement et intrinsèquement associées à des marchés et à des régulations nationales et, de ce fait, particulièrement rétives aux logiques d’intégration européenne et, a fortiori, de globalisation.

La décennie 90 a correspondu à une remise en cause de ces spécificités et les réductions des dépenses et réformes des modes d'acquisition des armes se sont alors opérées sous l'empire d'une conviction forte et partagée selon laquelle il était possible et souhaitable de "banaliser " (Sachwald, 1999) ou de "civiliser " (Barnier, 1996) ces activités. En 2009, vingt ans après la chute du mûr, la réalité de ce processus de "civilisation " commence à être mesurée et qualifiée. Certains travaux récents ont ainsi pu montrer que si d'importantes restructurations ont bien été générées par les changements qu'ont induit ces velléités, le produit scalaire des vecteurs d'inertie et des vecteurs de réformes dessine une orientation du secteur qui, pour l'essentiel, restaure une forme d'exotisme et confirme en particulier le "lien symbiotique " entre Etats et producteurs d'armes (Moura, 2007).

Cet article entend se pencher sur ce processus de changement malaisé en mobilisant une recherche conduite au début des années 2000 sur les politiques régionales d'aide à la reconversion

1 GREThA UMRS CNRS 5113, Université de Bordeaux, Avenue Léon Duguit, 33608 PESSAC Cedex, jullien@u-bordeaux4.fr

2 GREThA UMRS CNRS 5113, Université de Bordeaux, Avenue Léon Duguit, 33608 PESSAC Cedex, frigant@u-bordeaux4.fr 
des territoires marqués par les industries de défense utilisant les fonds européens dédiés à cet objectif appelés fonds Konver. Il s'était alors agi de reconstituer à la fois les trajectoires des régions concernées et de saisir le rôle que les politiques publiques y avaient tenu. Ce prisme particulier permet a posteriori de saisir la nature profonde du processus de restructuration des industries de défense tel qu'il s'est opéré dans cette phase clé.

Nous allons en effet montrer en mobilisant ce matériau que, au-delà des différences des trajectoires des systèmes productifs territoriaux (I) et du rôle tenu dans leur détermination par les politiques publiques régionales (II), c'est bien la reconstruction identitaire d'une industrie de défense toujours aussi peu banale qui s'opérait (III). Nous mobiliserons pour cela une grille d'analyse sectorielle institutionnaliste qui permettra, sur un plan plus général, de faire ressortir la place des politiques publiques régionales et européenne dans ces reconfigurations et de comprendre pourquoi, dans ce cas là, il fût marginal.

\section{L'inflexion des trajectoires productives: entre hétérogénéité et difficultés}

Les analyses des industries de défense ont depuis longtemps montré que leur émergence et leur développement est le fruit d'une imbrication étroite entre les sphères politique, économique et militaire. Les notions de complexe militaro-industriel, de mésosystème d'armement (Chesnais, Serfati, 1992), de mode de régulation administrée (Hébert, 1995) furent ainsi développées pour décrire un mode de fonctionnement où, finalement, c'est l'existence d'un lien symbiotique entre le monde industriel et l'Etat (au sens de la sphère politico-militaire) qui est affirmée.

Dans cette perspective, la décennie quatre-vingt-dix paraît rompre les équilibres antérieurs (Hébert, 1995). Les industriels de la défense se trouvent déstabilisés, notamment le secteur aéronautiquespatial-défense (ASD) sur lequel nous nous focaliserons. Le phénomène est général bien qu'il existe des différences nationales selon que le lien symbiotique qui unissait $1^{\prime} E$ tat $^{3}$ aux entreprises productrices d'armement était historiquement fort (Royaume-Uni et France) ou plutôt faible (Italie). Cette déstabilisation trouve sa source dans la conjonction de deux processus qui s'interpénètrent:

${ }^{3}$ L'Etat est la fois client car destinataire principal des productions et il définit le cadre légal et les aides aux exportations, industriel car producteur et sponsor car financeur de la RD et des développements préindustriels (Pour une analyse récente, $c f$. Moura, 2007).

140 Economie et Institutions - n²12\&13-2008-2009 
- une réduction des dépenses budgétaires et une réaffectation des crédits préservés sous le poids de l'effondrement du bloc de l'Est et la baisse des dépenses globales d'armement subséquente, de la première guerre du Golfe qui souligne l'importance des technologies de l'information comme outil majeur des guerres modernes et aboutit à une redéfinition des priorités entre programmes ;

- l'affirmation de la doctrine libérale dont un symptôme est la recherche de réduction des dépenses publiques mais qui plus largement conduit à repenser les modes d'acquisition.

Si ces transformations impulsées par les réformes entreprises nationalement renvoient en premier lieu à des questions de politique industrielle, elles soulèvent également des enjeux d'aménagement du territoire. En effet, l'industrie de défense se structure spatialement sur la base de bassins régionaux très marqués par les industries de défense et spécialisés, la plupart du temps, dans un type d'activité donné. Ainsi, l'Aquitaine, la région du North-West et la Lombardie se présentent comme des centres majeurs d'une production aéronautique-spatiale-défense singulièrement touchée. Sans en refaire l'historique, on peut rappeler que les facteurs de localisation initiaux des industriels dans ces trois régions relèvent essentiellement de considérations stratégiques et politiques qui, couplées à l'importance des coûts irrécouvrables et aux besoins en main d'œuvre hautement qualifiée, ont contribué à ancrer de grands établissements industriels dans ces territoires (Jalabert, 1974; Beckouche, 1996). Parallèlement, les contraintes et opportunités productives (taille des séries, faiblesse de la primauté du critère de coût, faible degré d'externalisation et recours massif au travail à façon...) et les modes de fonctionnement des relations interfirmes (faiblesse de la formalisation contractuelle, rôle des ingénieurs de production dans le choix des sous-traitants, culte du secret...) ont suscité la création de tissus denses de sous-traitants travaillant essentiellement pour les donneurs d'ordres locaux et ont ainsi généré de véritables systèmes productifs territorialisés - SPT (Frigant, Jullien, 1997 ; Economic Geography, 1993). Dans ce contexte, l'entrée en crise de l'industrie se traduit certes par des difficultés chez les donneurs d'ordres mais elle interroge plus encore le devenir de leurs preneurs d'ordres comme en témoignent les analyses et rapports statistiques établis dans les trois régions étudiées (CRCI-A, 1994 ; GSAD, 1996 ; LCC, 1994).

Face à l'ampleur de la crise, les industriels se laissent convaincre qu'il convient de sortir du militaire ou du moins de réduire leur dépendance envers la défense. Ceci leur apparaît d'autant plus nécessaire que la doctrine qui voulait que les dépenses

141 Economie et Institutions - nº 12\&13-2008-2009 
militaires aient, à terme, des effets positifs pour les activités civiles est elle-même contestée. Le paradigme des retombées laisse alors place au paradigme de la dualité inversée dès lors que l'on considère alors que ce sont désormais les activités civiles qui doivent nourrir les programmes militaires. De plus, les réformes des organismes d'acquisition convergent pour durcir les règles de surveillance sur les éventuelles rentes de situation et s'engage un processus - plus ou moins achevé (Kirat, Bayon, 2006) - qui prétend remettre en cause la prégnance du critère de performance technologique au profit de celui $\mathrm{du}$ coût d'usage (acquisition et maintien en conditions opérationnelles). On avait là autant d'éléments qui suggèraient que rester dans le militaire nécessitait malgré tout de se diversifier vers le civil.

Evidemment selon les activités réalisées et les caractéristiques des entreprises ou établissements (taille, appartenance à un groupe...), le mouvement ne pouvait être uniforme. Il ne le sera effectivement pas. Notre enquête faisait ainsi ressortir quatre trajectoires co-existantes.

Une première stratégie visait la diversification. Il s'agissait alors, sans renoncer aux activités de défense, de développer les activités civiles. Les entreprises concernées estimaient qu'elles pouvaient se positionner compétitivement sur les marchés civils car elles disposaient de compétences technologiques fortes développées dans un secteur de la défense très exigeant à cet égard. Sur cette base communément partagée, deux trajectoires se dessinent avec le recul. En premier lieu, quelques entreprises ou groupes réussissent effectivement à jouer la carte de la dualité. Il s'agit d'entreprises pour lesquelles les activités civiles visées sont proches des activités militaires. Par exemple, en Aquitaine, la société de maintenance aéronautique Sogerma parvient à accroître sensiblement la part de ses activités civiles. De même, Dassault se renforce dans les programmes civils grâce au succès du programme d'avion d'affaire Falcon. Toutefois, à côté de ces succès, ce qui domine, c'est plutôt l'échec de cette stratégie. Que ce soit d'ailleurs dans les grands groupes ou dans les PME. Trois séries de raisons expliquent cette deuxième trajectoire.

Les entreprises se heurtent à un problème d'adaptation des compétences organisationnelles. Les routines - au sens évolutionniste du terme (Nelson, Winter, 1977) - requises pour exceller dans la production d'armement s'avèrent assez radicalement différentes de celles du civil. Dès lors que les firmes cherchent à s'éloigner de leur cœur de compétences, elles se heurtent à de lourdes difficultés pour modifier leur manière de concevoir leur produit et inverser le sens des priorités entre critères de coûts et critères de performance

142 Economie et Institutions - $\mathrm{n}^{\circ} 12 \& 13-2008-2009$ 
technologique (Jullien, 1995). De même, les exigences en matière de management des programmes (par exemple le respect des délais) sont souvent énoncées par nos interlocuteurs comme une pratique difficile à diffuser au cœur de l'organisation. Enfin, la plupart des entreprises avaient manifestement sous-estimé combien les fonctionnements des marchés militaires étaient idiosyncrasiques. Là encore infléchir les routines des équipes commerciales s'avère compliqué.

Les structures industrielles et organisationnelles doivent s'adapter. Ce problème se retrouve principalement chez les PME car se diversifier oblige à dupliquer les processus de production. Travailler encore pour la défense oblige à conserver des structures dédiées (bureau d'études certifié défense; personnels affectés à ces activités...) et des processus orientés vers des petites séries. Dès lors, se diversifier conduit à mettre en place une structure quasiment parallèle. Par exemple, plusieurs PME lombardes s'engageant dans la fabrication de pièces pour automobile découvrent qu'elles doivent renouveler leurs équipements pour accéder à ce marché, telle entreprise nous explique qu'elle doit discriminer ses salariés selon civil/défense car les qualités individuelles requises sont différentes dans les deux mondes... En fait, si les savoirs semblent proches, et ont justifié l'intuition qu'il était possible d'aller vers le civil, les pratiques productives demeurent fondamentalement différentes et beaucoup de PME font l'amère découverte des difficultés qu'il y a à dualiser son activité à structures données.

Dans le prolongement direct, la dualité pose également - dans les groupes essentiellement - un problème de financement dans le sens où il convient de procéder à des arbitrages dans l'allocation des ressources. L'engagement dans des programmes civils suppose des avances de fonds dont les groupes n'avaient pas forcément l'habitude et exige surtout une pérennité des engagements (Lazonick, Prencipe, 2005). Celle-ci s'avère assez vite problématique pour certaines directions, faute de résultats à court terme, et des conflits émergent entre différentes options.

Pour résumer, le travail d'enquête montre combien il est difficile de sortir de la contrainte de sentier et de prétendre faire coexister - sur le plan technologique comme sur le plan organisationnel - deux logiques de fonctionnement parallèlement. De nombreuses expériences de diversification vont ainsi se solder par un recentrage sur la défense au terme de tentatives parfois coûteuses qui iront souvent jusqu'à mettre en danger la survie de PME qui avaient tenté de développer des produits propres pour sortir de la sous-traitance pour la défense.

143 Economie et Institutions - n²12\&13-2008-2009 
Cette difficulté se perçoit, par contraste, dans deux autres trajectoires plutôt couronnées de succès bien que très différentes. La première d'entre elle consiste à sortir radicalement du champ de la défense. Profitant des opportunités de financement public encourageant la reconversion externe ( $c f$. infra), certaines entreprises opèrent une véritable mutation sectorielle. A l'inverse, formant la quatrième trajectoire, d'autres entreprises font le pari de s'ancrer dans la défense. Cette stratégie s'appuie sur deux arguments. Le premier, opportuniste, part du constat que la disparition de concurrents (par faillite ou par choix de sortie de la défense) ouvre des opportunités de croissance même si les budgets globaux se réduisent. Le deuxième, essentiellement représentée chez les soustraitants, part du constat que les modifications du mode de fonctionnement des politiques d'acquisition et ses répercussions chez les donneurs d'ordres (accroissement de la pression sur les coûts, généralisation d'une contractualisation marchande se substituant aux pratiques informelles et à la confiance comme mécanisme de régulation de l'échange), conduisent à une "professionnalisation " du secteur où seules des firmes spécialisées et structurées selon les nouveaux principes organisationnels pourront réussir en jouant notamment la carte de l'internationalisation et de la diversification du portefeuille clients.

Ces quatre trajectoires concomitantes appellent deux commentaires. Tout d'abord, elles confirment combien les modes de fonctionnement de l'industrie d'armement sont spécifiques et les contraintes de sentier lourdes à lever. Ensuite, la comparaison interrégionale souligne que, si on peut repérer une certaine diversité au sein de chacune des régions, on peut y repérer des régularités. L'analyse micro-économique des processus de décision qui fondent la décision du dirigeant d'entreprise montrent que trois groupes de facteurs, internes et externes ${ }^{4}$, se conjuguent pour forger le schéma d'interprétation du spectre des possibilités parmi lesquelles le dirigeant tranche; jamais seul mais en lien avec ses clients principaux et les acteurs publics (Frigant, Moura, 2004). Compte tenu de la lourdeur des contraintes de sentiers, ce système public occupera un rôle souvent majeur pour inciter les firmes à jouer la carte de la diversification. En effet, face à l'ampleur de la crise, la période s'accompagne d'une forte créativité institutionnelle dans

4 Le processus décisionnel découle du croisement des représentations des dirigeants, de la contrainte de sentier découlant des compétences internes et de la contrainte de financement. Ces trois éléments sont infléchis par les discours et les mesures déployés par les donneurs d'ordres et les acteurs institutionnels.

144 Economie et Institutions - $\mathrm{n}^{\circ} 12 \& 13-2008-2009$ 
chaque région étudiée, et, sans être parfaitement déterministe, les dispositifs institutionnels mis en place jouent un rôle actif dans le processus de définition des trajectoires mais aussi de leur validation lorsque des moyens financiers existent. Toutefois, si l'enjeu partagé de ces dispositifs semble bien être celui d'encourager la reconversion, les traductions spécifiques données à cet objectif général sont assez disparates et le contenu même du terme reconversion s'avère dès lors assez versatile alors même que nos trois régions mobilisent un même programme européen visant explicitement la reconversion.

\section{L'appropriation contrastée de la problématique de la reconversion par les Systèmes locaux d'actions publiques}

Dès le tout début de la décennie, la Commission européenne s'inquiète des conséquences de la crise sur les bassins régionaux et elle initie un programme d'aides régionales dénommé Konver (I pour la période 1993-95 doté de 130M ECU, II pour la phase 1995-97 à hauteur de $500 \mathrm{M} \mathrm{ECU}$ ) dont la finalité vise clairement la sortie de la production d'armement pour les zones géographiques les plus affectées (les critères d'éligibilité sont établis sur la base du volume d'emplois perdus ou à perdre). L'intervention de la Commission est doublement intéressante. D'une part, elle constitue un contournement de l'article 223 du Traité de Rome qui interdit à l'Union de s'ingérer dans les domaines de la Défense; l'Union va ainsi s'occuper, non pas de la restructuration des industries de défense mais des conséquences de ces restructurations. D'autre part, son intervention a pour effet d'orienter le débat public vers la question de la reconversion externe au sens de sortie de la défense ou de diversification, par contraste avec la notion de reconversion interne où il s'agit de rester dans la défense et d'étendre le portefeuille clients ou la gamme des produits réalisés.

Les sommes mobilisées et la clarté des objectifs vont faire de l'échelle européenne un niveau important de définition des politiques publiques dans cette période. Toutefois, les dossiers Konver sont par définition instruits et portés par un niveau local (régional en l'espèce) de sorte que les dispositifs publics offerts aux industriels de la défense s'avèrent d'emblée multiniveaux. Européen par les fonds Konver, régionaux car cet échelon instruit les dossiers et peut en outre porter ses propres actions, nationaux car chaque Etat entend conserver un droit de regard sur ces industries éminemment stratégiques.

De fait, si la "puissance publique " est bien agissante face à la crise et infléchit les décisions des industriels, ce terme cache une pluralité d'acteurs et, au niveau régional, l'intervention publique va renvoyer à des processus et des contenus disparates parce que les interventions et leurs objectifs sont dissemblables et parce que les

145 Economie et Institutions - $\mathrm{n}^{\circ} 12 \& 13-2008-2009$ 
problèmes productifs mis en avant par les industriels apparaissent différents. D'un point de vue méthodologique, le travail d'enquête qualitatif auprès de l'ensemble des acteurs impliqués, permet toutefois de percevoir que des formes de cohérence se dessinent dans les jeux interactifs multi-niveaux et pluripartites. On peut ainsi caractériser un Système Local d'Action Publique (SLAP) que l'on peut définir comme la concrétisation du jeu des acteurs multi-niveaux porteurs de logiques qui ne sont pas forcément cohérentes ex ante mais qui finissent par former une politique dont l'objet devient finalement possible à interpréter par des observateurs externes. De fait, trois SLAP forts distincts s'élaborent dans chacune des régions.

\subsection{Une impulsion privée relayée par les acteurs publics dans le North West : une reconversion interne}

La crise de l'industrie de la défense affecte une région encore fortement marquée par les séquelles de la crise de la métallurgie. Dans ce contexte, les dangers de la réduction des dépenses d'armement sont très précocement saisis par les acteurs locaux et aboutissent dès 1992, à la création d'une association visant à " promouvoir à la fois la création et le maintien des emplois " (website de la NWAA, page d'accueil en 2001). La North West Aerospace Alliance (NWAA) est constituée par les industriels de l'aérospatial et se présente elle-même comme une organisation de lobbying visant à assurer le développement de l'industrie ASD locale. Elle regroupe plus de 200 membres allant des donneurs d'ordres (Rolls-Royce, BAE System...) à tout un ensemble de PME travaillant directement ou indirectement pour l'ASD.

C'est essentiellement autour de cette association que l'action publique régionale se structure. Les objectifs affichés par la NWAA sont de combiner les ressources locales afin d'accroître d'une part le potentiel technologique des PME, en organisant leurs regroupements et en facilitant les transferts de compétences techniques et organisationnelles entre les membres, et d'autre part leur offre commerciale en particulier à l'exportation. Dans cette perspective, la NWAA se montre très active en matière de participation aux salons aéronautiques et a tissé des liens avec près de 23 associations ou organismes locaux professionnels, plus ou moins liés à l'ASD, relevant de 11 pays différents. Bien que l'association encourage officiellement ses membres à se diversifier, son objectif principal est d'assurer l'ancrage viable de ses membres dans le secteur ASD. Dit autrement, la NWAA privilégie les soutiens qui visent à conforter le positionnement des entreprises locales dans l'ASD. L'objectif principal de l'association est de promouvoir une reconversion interne, dans le sens où il s'agit essentiellement de renforcer la compétitivité du système productif territorialisé dans la production d'armes.

146 Economie et Institutions - n² $12 \& 13-2008-2009$ 
Cette stratégie territoriale recouvre celle adoptée par BAE System. Cette correspondance n'est sûrement pas fortuite lorsqu'on prend acte de ce que le directeur de la NWAA, John Whalley, travaille pour le constructeur d'avions de combat, par ailleurs principal employeur ASD de la région. Le rôle dominant de la NWAA se renforce par l'étroite connexion qu'elle possède avec l'acteur public régional.

Bien que plus tardive, la décentralisation au Royaume Uni a conduit à créer un pôle institutionnel en région autour de la formation de "gouvernements locaux". Le Government Office for the North West (GONW), constitué en 1994, dispose de prérogatives importantes en matière de politique économique qui deviendront effectives en 1998. Il définit notamment en lien avec les autorités nationales, la nature des interventions économiques à promouvoir à l'échelle de la région. Il a ainsi la responsabilité de mettre en œuvre localement les actions définies par les ministères, notamment les programmes "d'assistances régionales" du département de l'industrie et du commerce (DDA, 2000). Bien qu'il puisse exister des passerelles entre les différents types d'aides auxquelles peuvent prétendre les entreprises, le principal vecteur de la politique de reconversion est l'Agence de Développement Régional. Si le positionnement de cette institution est plus difficile à identifier, il semble qu'elle limite ses interventions en faveur d'une sortie de l'armement. Un indice probant de ce positionnement est que le directeur de la section aérospatiale est $\mathrm{J}$. Whalley, directeur de la NWAA, dont on voit mal qu'il infirme la politique prônée par l'association au niveau régional.

C'est finalement par l'échelon national que s'affirme l'objectif le plus clair en faveur de la diversification en dehors de l'armement (reconversion externe). Dans sa plate-forme électorale, le parti travailliste avait annoncé son intention de soutenir son industrie de la défense et promis la création d'une agence de diversification. En mars 1998, ce projet prend corps avec la publication d'un green paper (MoD, 1998). Placée sous le contrôle de la DERA (Defence Evaluation and Research Agency), la Defence Diversification Agency (DDA) a pour objet :

- de promouvoir l'exploitation des technologies militaires vers des applications civiles ;

- d'encourager la diversification des firmes dépendantes des programmes d'armement que ce soit vers le civil ou en termes d'accès à de nouveaux marchés militaires à l'exportation ;

- de favoriser l'utilisation de technologies civiles vers les industries de la défense.

Néanmoins, le manque de mesures précises adoptées par la DDA n'aboutira pas durant cette décennie 90 à orienter clairement

147 Economie et Institutions - n²12\&13-2008-2009 
les acteurs industriels dans cette voie de la diversification, et ce d'autant moins dans le North-West où l'agence locale n'est toujours par installée en 2000. De fait, l'ensemble de la décennie se résume plutôt par une conception de la reconversion laissé à ses parties prenantes essentielles : les industriels.

Marqués par les précédentes crises locales, les acteurs locaux ont rapidement réagi par le biais initialement des acteurs privés relayés par la suite par l'action publique. Dominé par la sphère industrielle, le compromis entre système productif territorialisé (SPT) et système local d'action publique (SLAP) se noue clairement en faveur d'une politique de sauvegarde des emplois, ce qui conduit à renforcer les liens de solidarité entre les firmes locales et à s'engager dans une recherche de compétitivité accrue dans l'industrie ASD. La relative absence de l'Etat central apparaît prégnante. C'est en particulier le cas de la DERA, qui se borne presque exclusivement dans un premier temps à se mobiliser pour inscrire la région dans le programme Konver, puis définit une stratégie de reconversion externe mais sans guère de conviction, localement du moins.

Ceci semble indiquer que le SLAP est intervenu de manière plutôt pro-active. Ses différentes composantes ont cherché par leurs interventions à favoriser l'adaptation des industriels régionaux aux nouvelles contraintes technologiques et commerciales. Elles n'ont par contre guère cherché pour les ressources constituées territorialement des domaines alternatifs de valorisation. En ce sens, on pourrait presque dire que même si des fonds Konver ont été mobilisés, il n'y a pas eu réellement dans cette région de processus de reconversion externe véritablement conçu. En ce sens l'exemple anglais s'oppose fortement au cas français.

\subsection{Le rôle essentiel des acteurs publics en Aquitaine : une reconversion ambiguë}

Si les acteurs publics sont apparus en relatif retrait dans la mise en œuvre des actions visant favoriser la reconversion de l'industrie britannique, en Aquitaine, ils vont occuper une place centrale. Cependant, deux périodes méritent d'être distinguées.

$\mathrm{Au}$ début de la décennie 90, les acteurs publics locaux prennent conscience de l'ampleur de la crise par le biais d'une série d'audits commandée par le Conseil Régional. Dans le même temps, le Conseil Economique et Sociale d'Aquitaine parvient à des conclusions tout aussi alarmistes (CESA, 1992). Cette prise de conscience entraîne une première série de réactions et notamment la création d'un Fonds Régional d'Aide à la Mutation des Industries de la Défense, de l'Armement et de l'Espace (FRAMIDAE) en 1992, complété par la suite par plusieurs autres initiatives plus restreintes. Parallèlement, au niveau national, le ministère de la Défense met en

148 Economie et Institutions $-\mathrm{n}^{\circ} 12 \& 13-2008-2009$ 
place le Fonds de Restructuration de la Défense (FRED) qui vise à atténuer les conséquences économiques et sociales des restructurations des industries d'armement. En outre, il charge en 1993 une société de conversion d'une mission de trois ans sur la zone d'emploi de Bordeaux dont les objectifs sont l'élaboration d'un diagnostic et d'un plan de revitalisation, la détection des projets créateurs d'emploi, le montage de dossier d'aides (FRED notamment) et l'accompagnement et le conseil des porteurs de projets. Cette première période apparaît avec le recul comme une période transitoire où l'ensemble des acteurs publics pris sous le feu de la violence de la crise cherchent à compiler les mesures sans véritablement définir une ligne stratégique précise. Les rapports se succèdent, des crédits sont alloués mais les mesures semblent essentiellement guidées par une volonté de sauver l'existant. Une caractéristique de cette période est l'absence d'un coordinateur majeur. Certes, la société de conversion tend à devenir l'acteur pivot de l'ensemble des dispositifs mais ceci reste tacite.

L'année 1995 constitue une rupture. Tout d'abord, la prise de fonction du Délégué régional au redéploiement industriel et aux restructurations de la défense marque l'introduction du coordinateur qui manquait au SLAP. Sa fonction est double 1) assurer la consolidation, le redéploiement et la diversification des industries de défense ; 2) animer l'accompagnement social des restructurations, la reconversion des sites et le reclassement des personnels publics. Ensuite, cette même année, la signature d'une convention EtatRégion cristallise l'évolution de la politique publique et fige l'architecture globale des modalités d'utilisation des diverses aides existantes (FRAMIDAE, Konver, FRED...). Cette convention comporte en elle-même les germes de l'ambiguïté de la reconversion. D'un côté, retrouvant la logique de sauvetage de la première période, une série d'objectifs vise à renforcer l'inscription ASD des grands établissements aquitains et de certaines PME aux compétences jugées stratégiques pour la défense nationale. De l'autre, une série d'objectifs vise à identifier et allouer des crédits concernant un vaste spectre de domaines pour permettre la diversification des PME. On a ainsi dans les objectifs une ambiguïté assumée qui laisse ouvert le processus d'élaboration d'un compromis.

La mise en œuvre de cette convention va confirmer cette ambiguïté. D'un côté, l'intervention publique s'inscrit dans une logique de conservation du système productif territorial dans sa vocation militaire, comme en témoignent plusieurs aides accordées à des " projets défense ". Au delà toutefois, c'est, plus généralement, la vocation aérospatiale de la région que l'on souhaite affirmer et soutenir comme l'illustrent les efforts réalisés pour soutenir le développement de la maintenance aéronautique bordelaise ou les tentatives d'obtenir une partie de la charge de travail liée à l'A380.

149 Economie et Institutions - n² $12 \& 13-2008-2009$ 
De l'autre, le délégué à la reconversion, après une évaluation des compétences des PME dresse une liste, sélective, d'entreprises aptes à s'engager en dehors de l'ASD. Cette action n'est cependant pas sans paradoxe. En effet, certaines entreprises convaincues de la difficulté à combiner une organisation bicéphale, tournée vers la défense et le civil, vont sortir définitivement du secteur ASD et lever ainsi l'ambiguïté que les aides nourrissaient. A contrario, certaines s'engagent dans un premier temps vers la recherche de nouveaux secteurs et cherchent à mettre au point des produits propres, mais sous l'effet de l'amélioration conjoncturelle de l'aérospatial civil, voire même la confirmation de certains programmes militaires (M51), renoncent à leur initiative de diversification. Ces échecs, regrettés par le délégué à la reconversion, soulignent que le processus de reconversion est bien le fruit d'un compromis avec l'ensemble des acteurs. Un point paradoxal est que fréquemment les entreprises qui ont le mieux réussi à se diversifier sont celles qui se sont le plus éloignées du système local d'action public, d'évidence limité dans ses compétences à favoriser l'émergence d'alternatives car porteur d'une ambiguïté fondamentale dans ses propres objectifs.

Contrairement au cas britannique, les acteurs industriels sont souvent restés en retrait dans le processus de reconversion. Dès la première période, Grossetti et al. (1994) soulignaient le manque d'implication des industriels dans le fonctionnement des fonds FRED. De fait, ce retrait de la sphère industrielle constitue un trait marquant de l'Aquitaine. L'existence d'une association regroupant les principaux industriels aquitains (BAAS), pouvant être rapproché sur le papier de la NWAA britannique, suggérait que des connexions entre les deux sphères industrielles et publiques auraient pu se nouer. Néanmoins, si des contacts existent, il manque une véritable implication des industriels dans le SLAP. Une des difficultés majeures à l'absence d'une politique claire en Aquitaine provient de l'insuffisance d'une véritable négociation sur le compromis à nouer entre le SPT et le SLAP. Certes certaines missions d'aides au développement aux PME des donneurs d'ordres font l'objet d'une concertation avec le délégué à la reconversion (Aérospatiale Développement par exemple) mais cela participe plus d'opérations ponctuelles qu'il s'agit d'ajouter à un catalogue d'aides déjà disparates.

L'Aquitaine se distingue donc par une incapacité à définir un compromis négocié entre les systèmes d'action publique et le système industriel. Il en résulte que l'intervention publique adopte une démarche d'aides tous azimuts, gérant les demandes dispersées des industriels, PME et grands établissements, dont les stratégies de survie face à la crise sont hétérogènes. Cette hétérogénéité conduit en retour l'intervention publique à osciller dans sa mission de reconversion qui alterne entre reconversion interne, promotion d'une

150 Economie et Institutions - n $12 \& 13-2008-2009$ 
présence duale militaire/civile et reconversion externe. $\mathrm{Si}$, en laissant ouvert l'espace du compromis, cette manière de faire pouvait au départ apparaître comme relativement judicieuse tactiquement, le fait qu'elle perdure sur la période semble indiquer que l'apprentissage institutionnel est quelque peu défaillant ce qui pourrait être la contrepartie du choix qui a été fait très tôt d'opter dans la recomposition du SLAP pour des dispositifs très " coordonnés " voire très centralisés.

\subsection{La Lombardie : d'un compromis national en faveur de la reconversion interne à l'échec d'un compromis local en faveur de la diversification}

Le cas italien correspond à une troisième configuration où le compromis entre le SPT et le SLAP s'est noué essentiellement au

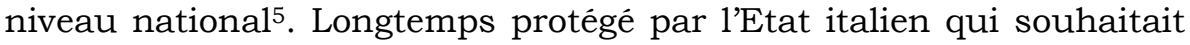
se doter d'une industrie performante ${ }^{6}$ les établissements lombards sont d'autant plus affectés par la baisse des dépenses d'armement qu'ils souffrent d'un manque cruel de capitalisation, de capacités de $\mathrm{R} \& \mathrm{D}$ et de normes de gestion bureaucratique. Leur réponse à la crise prend alors la forme d'une reconversion interne. La priorité est de restructurer les activités en modernisant l'organisation productive afin d'accroître leur compétitivité en restant dans le champ étroit de l'armement, à l'exception d'Aermacchi qui cherche à dualiser sa production aéronautique vers le civil. Ceci se traduit par d'importants plans de licenciement qui trouvent un premier aval de l'Etat central par la loi du 23/07/1991 qui prévoit des mesures pour favoriser et traiter socialement les conséquences des plans de restructuration.

Cette phase purement défensive fait place à partir de 1993 à une seconde dont le discours se veut plus prospectif. En 1993, le gouvernement adopte une loi visant à faciliter la rationalisation, la conversion vers le civil et la dualité civil/militaire des industries de la défense. Néanmoins, l'affichage en faveur de la diversification ne se traduit pas dans les faits. Un bilan réalisé en 1998 révèle que 80\% des fonds octroyés au titre de cette loi furent affectés à la restructuration et la consolidation de l'industrie de la défense, et seulement $20 \%$ pour la conversion. Le ministère de l'industrie en charge de ces fonds privilégie en effet les projets de R\&D militaire ASD et l'aide à l'exportation d'armement. La loi suivante de 1994 prolongera cette logique de reconversion interne en promouvant les

5 Ce paragraphe s'appuie essentiellement sur G. Perani (2000) et Graziola G., Parazzini S. (2001).

6 En témoigne la loi de soutien au secteur aéronautique en 1985 qui sera suivie de plusieurs lois de refinancement.

151 Economie et Institutions - n²12\&13-2008-2009 
coopérations internationales et l'amélioration de la compétitivité des firmes militaires.

Cette absence de mobilisation du gouvernement central en faveur de la diversification trouve son point d'orgue dans son absence d'implication dans le programme Konver. Ne percevant pas l'utilité de Konver I, le gouvernement n'initie aucune action pour y souscrire. Face à la protestation de certains parlementaires, le gouvernement change brutalement sa position et, tout en refusant d'en prendre l'initiative, demande aux régions de lui soumettre des projets qu'il leur promet de soutenir auprès de la commission. Cette démarche explique que seulement quatre provinces dépendant de quatre régions bénéficient de ce programme dont les projets dénotent d'une absence de vison stratégique et de cohérence productive. Si la Lombardie n'appartient pas à ce premier Konver, elle s'engage par la suite fortement dans le second en essayant de rompre radicalement avec la logique de sauvetage des activités d'armement de l'Etat central.

En effet, la région lombarde crée, en 1994, une agence régionale de conversion dont la mission vise à "foster the creation of new entrepreneurial activities using military technologies in civilian fields (...) linked with the chance for synergies offered in Lombardia by implementing of the Konver programm (...) These two instruments could offer relevant opportunities for development of military firms in Lombardia " (Regional Law of Lombardia, 1994). Exemple unique en Italie, la région adopte donc une loi qui entend explicitement inciter les industriels à sortir du militaire. Deux missions sont attribuées à l'agence: promouvoir toutes les initiatives visant la conversion productive du militaire vers le civil d'une part, et soutenir des projets spécifiques qui contribuent à assurer des transferts technologiques vers le civil d'autre part. Pour parvenir à ces fins, l'agence cherche à s'assurer la participation de l'ensemble des acteurs locaux en intégrant dans son conseil de direction des représentants de la région, des associations d'industriels, des syndicalistes et des chercheurs en économie industrielle. En outre, pour accroître sa force d'action, elle cherche à centraliser l'ensemble des aides potentielles qu'elle prétend coordonner. Son budget doit en effet s'appuyer sur une large collecte des fonds régionaux, des aides nationales et des fonds européens, principalement Konver.

Le bilan de l'agence apparaît contrasté avec le recul. Si certains projets semblent effectivement des succès, son action fut cependant trop brève et les dérives constatées empêchent d'y voir une pleine réussite. En 1996, les activités de l'agence sont suspendues. L'explication tient en fait à l'incapacité dont elle a fait preuve à sédimenter un compromis local autour de son objectif de reconversion. Trois niveaux sont en cause. 
Le premier concerne le compromis entre les acteurs locaux du SLAP qui ne se faisant pas conduit à son éclatement. Les élections conduisent en effet à la tête de la région une majorité de droite qui s'avère rapidement hostile à la politique de conversion. Arguant que la priorité n'est pas à assurer la diversification de son industrie de défense, la région retire ses représentants du conseil de direction et réduit fortement les fonds octroyés à l'agence. Cette dissolution du compromis local est amplifiée par l'attitude des industriels. A un deuxième niveau en effet, ces derniers se montrent, dans leur majorité, réticents à la politique de conversion. Des dérives sont notées dans l'utilisation des crédits accordés par l'agence : certaines entreprises profitent de l'effet d'aubaine pour s'engager dans des pseudo-projets de conversion qu'elles ne mènent pas à terme. Cette difficulté à mobiliser les industriels en faveur de la diversification s'explique en partie par l'action même de l'Etat central (troisième niveau) qui ne s'insère pas dans le compromis local proposé par l'agence. Durant la période, l'Etat poursuit sa politique visant à pérenniser son industrie d'armement. L'asymétrie des moyens financiers entre l'agence régionale et l'Etat contribue à infléchir l'attitude des industriels. Cette insuffisance des crédits tient en partie à une erreur même de positionnement stratégique de l'agence vis-à-vis de l'institution européenne. L'agence espérait mobiliser largement les fonds Konver. Néanmoins ces fonds s'accordent mal avec ses propres objectifs puisqu'ils privilégient les PME, le développement régional et ciblent des zones précises.

L'expérience de l'agence régionale de conversion lombarde illustre bien que le succès d'une réelle politique de conversion ne peut se réaliser que si l'ensemble des parties prenantes à la reconversion s'accorde sur l'objectif à atteindre. L'absence d'unanimité au niveau politique local, la réticence des industriels régionaux à quitter l'armement auquel fait écho l'Etat central qui les encourage dans cette voie et les difficultés à harmoniser les aides européennes aux objectifs de l'agence sont autant de causes d'échec qui illustre qu'une stratégie claire de reconversion nécessite l'adhésion de toutes les parties. De fait, le seul compromis qui semble s'être fait jour en Lombardie est celui en faveur de la reconversion interne à partir de l'action de l'Etat central soucieux de préserver son industrie ASD et des industriels favorables à leur spécialisation dans ce domaine productif.

L'examen des dispositifs publics s'instaurant dans les trois régions souligne, en dépit d'une thématique partagée dans les discours en termes de "nécessité de se reconvertir face à la crise " et d'une opportunité réelle offerte par l'initiative Konver, régionalement, que ce sont des compromis spécifiques, construits selon des processus idiosyncrasiques tenant à l'histoire des régions, aux

153 Economie et Institutions $-\mathrm{n}^{\circ} 12 \& 13-2008-2009$ 
industriels présents et capables (ou non) de s'insérer dans le jeu public, et, au niveau des acteurs publics selon la manière dont s'élabore l'interaction nationale/locale, qui finalement se nouent pour forger chaque système local d'action publique. De fait, à ce stade de l'analyse, notre travail permet de comprendre en partie comment s'articulaient les trajectoires des systèmes productif territorialisé et public local. Toutefois, des zones d'ombres demeuraient dans notre analyse concernant les trajectoires sectorielles. Pour les saisir, il est en effet nécessaire de dépasser les approches micro-économiques déployées dans la première section.

\section{Une relecture institutionnaliste des dynamiques sectorielles et régionales}

La difficulté à échapper durablement à la contrainte de sentier institutionnelle apparaît dans nos trois cas telle que, avec des trajectoires différentes, c'est toujours l'option défensive qui finit par prévaloir. Toutefois cette défense est partout ambiguë et laisse à l'émergence d'alternatives productives des chances inégales : celles ci sont minimales dans le cas anglais, plus importantes dans les cas français et italiens mais l'avenir de ces régions et leurs chances de demeurer demain des grandes régions productrices du secteur ASD reste incertain dès lors que l'ambiguïté fondamentale n'apparaît toujours pas avoir été levée sur le contenu à donner à la reconversion.

Les SLAP ressortent ainsi comme soumis à des logiques de restructuration qu'ils ne maittrisent pas et sur lesquels ils ne peuvent intervenir qu'à la marge. Dans leur structuration et leur positionnement la question fondamentale peut se synthétiser dans l'opposition pro-activité/contra-activité. L'examen des cas semble indiquer que le caractère régional - c'est-à-dire fondamentalement second - des interventions invalide les prétentions contra-actives. La posture contra-active consiste à tenter d'impulser localement une logique de restructuration qui propose ou impose aux entreprises des réorientations de leurs activités productives contradictoires avec celles que semblent imposer les évolutions des industries de défense. La posture pro-active consiste pour les acteurs du SLAP à considérer qu'ils n'ont pas prise sur les dites évolutions et qu'ils ne peuvent imposer aux entreprises des alternatives. Dès lors, c'est à accompagner les entreprises à rester dans l'ASD et - éventuellement - à s'y développer que l'on va s'employer. La première posture est justifiable sur le papier mais difficilement tenable face aux entreprises qui, refusant de lâcher la proie pour l'ombre, vont avoir tendance à ignorer les interventions du SLAP. C'est celle qu'avaient retenue les autorités locales lombardes dans un premier temps. La seconde posture parait plus réaliste mais elle pose la question de

154 Economie et Institutions - n²12\&13-2008-2009 
savoir si les évolutions constatées ne se seraient pas produites en l'absence d'aides, si, en d'autres termes, elle ne se contente pas de créer des effets d'aubaine. L'exemple britannique et sa respécialisation dans l'ASD au service du champion national BAe illustre ce cas de figure.

Le cas aquitain dans lequel s'est dégagé pendant un temps un dispositif assez coordonné d'interventions publiques peut s'interpréter comme la mise à jour d'une espèce de troisième voie. Elle a consisté au fond à refuser de choisir entre une et l'autre option: en maintenant cette ambiguïté, le DRIRD renforce politiquement sa faculté de coordination en n'offrant à aucune composante du SLAP de raisons de s'affranchir de ses orientations ; il donne également aux entreprises la possibilité de s'adosser aux aides à la reconversion pour se respécialiser, se diversifier ou quitter l'ASD. Là où, de fait, la logique exogène de restructuration de l'ASD semble imposer une espèce de tout ou rien, le SLAP feint de considérer que la diversification est possible. Elle l'est effectivement s'il s'agit de celle du tissu productif et non celle des entreprises. Il suffisait de ne pas le dire pour aider à ce qu'elle s'opère.

Les trois cas étudié témoignent de la très grande "plasticité " des dispositifs d'aide Konver qui d'évidence ont pu être mobilisé pour s'inscrire dans des perspectives stratégiques fort différentes et fort changeantes. D'une certaine manière, l'ambiguïté que nous décrivons pour le cas aquitain est aussi celle de la politique européenne en la matière. Elle renvoie au fait que dans un cas comme dans l'autre le processus fondamental à l'œuvre est hors du champ de compétences de l'UE comme des composantes des SLAP qui se contentent d'en gérer les effets en essayant chemin faisant d'en saisir la teneur. De ce point de vue, on peut avancer l'idée qu'il y a eu dans cette période une asymétrie grandissante entre l'UE et les SLAP dans la mesure où les SLAP comme lieu d'apprentissage institutionnel ont eu dans cette période à statuer sur le bien-fondé et/ou la performativité des principales hypothèses de travail des réformateurs. La possibilité de dualiser par exemple est devenue pour les acteurs des SLAP une question opérationnelle à laquelle ils ont contribué à répondre par leurs interventions. Leur histoire est donc aussi celle de la restructuration de l'ASD. Elle montre que, fondamentalement, le cœur identitaire de cette industrie a continué de battre malgré les réformes.

En mobilisant un cadre d'analyse sectoriel que nous exposerons rapidement au préalable (III.1), nous allons, pour clore cette analyse, pratiquer cette troisième entrée. Nous pourrons alors mieux interpréter en quoi ont consisté les aides aux reconversions décrites (III.2). 


\subsection{La structuration du champ concurrentiel}

Pour saisir les identités sectorielles et leurs éventuels changements, nous avons développé une représentation des industries en les interprétant comme des champs concurrentiels (Jullien, Smith, eds, 2008). Nous traitons alorsles-dits champs comme des espaces régulés dans lequel les incertitudes sont réduites à la fois par des références communes et un certain mimétisme, et par une action collective qui amène les firmes à définir conjointement les conditions dans lesquelles elles collaborent et/ou entrent en rivalité. Pour cela, on les représente comme inscrites dans cinq sphères : celle de la production et de la concurrence (au cœur du schéma) et quatre sphères liées qui correspondent aux conditions dans lesquelles elles accèdent aux 4 grandes catégories de ressources que sont les financements, les approvisionnements, le travail et les clients et dans lesquelles le couple concurrencecoopération se trouve projeté en même temps que soumis à des contraintes externes qui renvoient à la place de l'industrie dans le système productif et social global. Ces quatre sphères font l'objet de régulations qui se spécifient et se stabilisent au niveau du secteur et que nous nommons " rapports institués " (RI).

\section{Figure 1 : Un champ concurrentiel structuré autour de 4 piliers}

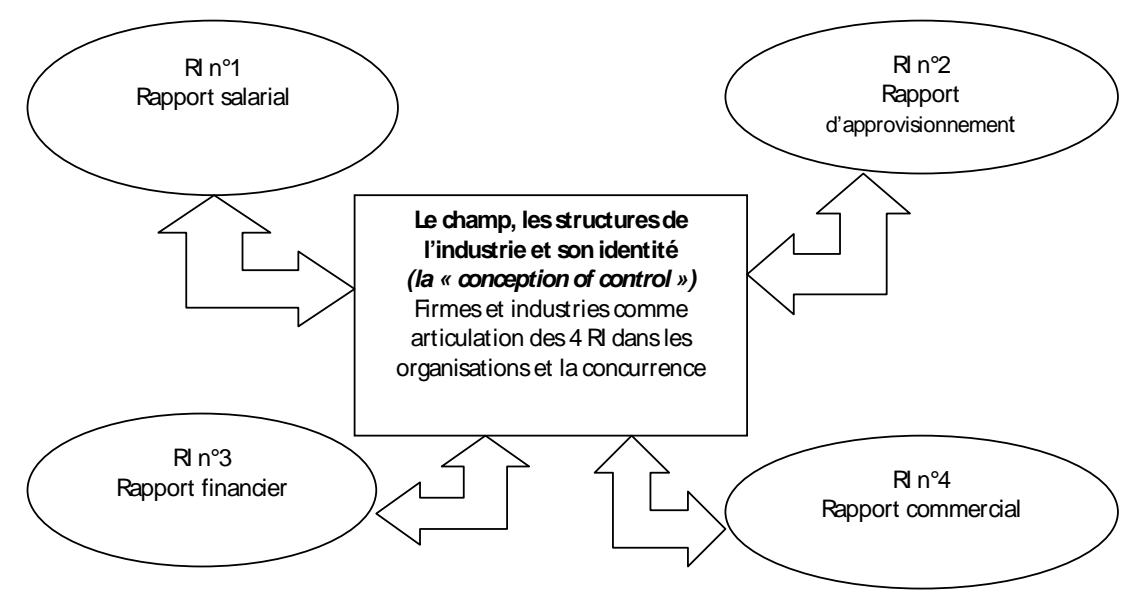

La production et la structuration de stratégies imposent d'articuler ces rapports. Cette articulation définit, quand on l'envisage au niveau de la population des firmes rivales, la structure du champ. Envisagée du point de vue de chaque firme, l'organisation en assure l'articulation en produisant une interprétation spécifique de chacun des rapports et en définissant ainsi un positionnement

156 Economie et Institutions $-\mathrm{n}^{\circ} 12 \& 13-2008-2009$ 
stratégique. Le champ est ainsi une forme d'accès spécifique aux quatre grandes catégories de ressources et un espace de différenciation des stratégies dans lequel les places occupées par les firmes sont distribuées de telle sorte que le "régime concurrentiel " y est " soutenable".

Pour traiter les questions d'identité sectorielle, de structures et de restructurations des industries, ce cadre permet de distinguer les différents niveaux de régulation. En effet, l'hypothèse est que les formes d'institution des différents rapports sont distinctes. De manière plus précise, notre cadre repose sur l'hypothèse que chaque rapport institué est susceptible d'avoir pour chaque industrie une structure spécifique, de se différencier pour une même industrie des autres rapports institués structurant du point de vue des normes juridiques applicables entre autres et enfin de se modifier et d'entraîner des restructurations plus ou moins amples de l'industrie selon qu'il " contamine " ou non les autres rapports institués.

Pour qualifier nos RI du point de vue de leur cohérence " interne ", nous proposons d'envisager la dimension sociale de la transaction que l'institutionnalisation du rapport doit régler dans deux registres à la fois distincts et liés : celui des relations entre détenteurs et utilisateurs des ressources et celui des relations entre concurrents. En effet, dès lors que nous définissons une industrie comme un ordre institutionnel qui est une manière dominante de concevoir la production et la concurrence dans une activité donnée, un espace donné et une période donnée, les RI ressortent comme dotés d'une double nature : il sont à la fois des relations entre "stakeholders " engagés dans la production et des relations entre concurrents projetant leur rivalité dans chacun des RI. C'est la raison pour laquelle les relations de pouvoir et la division des rôles entre dominants et dominés dans le champ que Fligstein met au cœur de sa définition des " conceptions du contrôle " sont envisagées dans notre grille d'analyse au niveau de chacun des RI.

Ces rapports sont donc à la fois rapports des dirigeants aux salariés, des dirigeants aux actionnaires, des acheteurs aux fournisseurs et des commerciaux ou concepteurs des produits aux clients et rapports de concurrence des firmes entre elles dans chacun de ces quatre registres. Ces rapports sont définis au niveau de l'industrie en lien les uns avec les autres puisque, ensemble, ils définissent un ordre qui doit dégager une complémentarité institutionnelle qui le dote à la fois de la fonctionnalité minimale et de la soutenabilité politique dont il a besoin pour être pérenne et

157 Economie et Institutions - nº $12 \& 13-2008-2009$ 
organiser ainsi, politiquement et économiquement, la vie de l'industrie? ${ }^{7}$.

L'intérêt de la représentation proposée tient au fait que les échelles géographiques auxquelles renvoient ces RI ne sont les mêmes ni d'une industrie à l'autre, ni d'une période à l'autre, ni d'un RI à l'autre pour une même industrie. On peut en effet considérer que quatre échelles territoriales doivent s'articuler dans l'élaboration des régulations : l'échelle des territoires, l'échelle nationale, l'échelle régionale (UE par exemple) et l'échelle globale. Le travail politique multi-niveau par lequel les acteurs se saisissent des opportunités qu'ils perçoivent à ces échelles détermine alors une prédominance de l'un ou l'autre niveau pour chacun des RI. On a alors le choix entre deux usages possibles de notre représentation. Le premier consiste à retenir le plus petit commun dénominateur et à définir l'industrie à ce niveau pour ne représenter au cœur du schéma que des acteurs soumis pour les quatre rapports aux mêmes contraintes. Le second consiste à retenir plutôt le plus grand dénominateur et à considérer la différenciation constatée aux différentes échelles territoriales comme dessinant des espèces de sous-secteurs en concurrence. Bien évidemment, ces deux approches sont complémentaires mais c'est plutôt la seconde qui semble indiquée lorsqu'il s'agit de s'enquérir du degré de globalisation des activités ou d'examiner la question des lieux de la concurrence ou des échelles géographiques pertinentes pour en restituer la dynamique.

\subsection{Une réinterprétation des trajectoires de reconversion}

Le cadre conceptuel présenté ici permet de cerner comment se posent les questions de reconversion des industries de défense relativement à ce que sont à la fois les structures de ces industries et la dynamique de restructuration des dites industries. Ainsi resituées dans le contexte sectoriel, les questions de reconversion des régions productrices apparaissent dans toutes leurs difficultés et les stratégies et "performances" des différents SLAP gagnent en lisibilité. In fine, le caractère très partiel de l'intervention européenne et la maîtrise, non moins partielle, de ses effets sont restitués. Se voient alors posées à travers nos trois cas le problème de la nature de l'intégration européenne lorsque l'on l'examine à travers la vie des industries.

\footnotetext{
7 Pour rendre compte des dimensions trans-sectorielles et montrer comment ce qui se noue comme RI est lié au reste de la société et de l'économie, nous proposons ailleurs (Jullien, Smith, 2008) une présentation plus complète de ces RI où, en surplomb de cette matrice, les ressources en provenance des ordres politique, juridique et économique prévalant globalement sont exhibées.
}

158 Economie et Institutions - n²12\&13-2008-2009 
Projeté dans notre cadre d'analyse, la dynamique fondamentale imprimée aux industries de défense à partir des années 90 peut se synthétiser en repartant de la thématique de la banalisation qui avait été introduite à l'époque par Sachwald (1999). Appelée également "civilisation", il s'agissait, a-t-on alors pensé, de faire des opérateurs de ces industries des entreprises "normales" qui auraient gagné en efficience en étant en concurrence et qui auraient dû pour cela accepter que se réinstitutionnalisent chacun des 4 RI jusqu'ici définis dans la logique très spécifique de l'arsenal. Ainsi d'abord, au niveau du rapport commercial, les armées devaient devenir de vrais acheteurs et bénéficier pour cela de services dédiés de leurs ministères qui auraient rompu leurs liens symbiotiques avec les industriels nationaux pour que le "smart procurement" puisse désormais être la règle. Ensuite, au niveau du rapport financier, il paraissait doublement nécessaire de modifier la nature du contrôle exercé sur les fabricants d'armes pour que la quête d'efficience devienne la règle. En privatisant, on soumettait en effet d'abord les entreprises à une quête de rentabilité qui n'avait guère de raisons d'être la leur antérieurement. On créait d'autre part un marché du contrôle qui devait permettre que s'opèrent, au niveau international y compris, les recompositions industrielles qu'appelait la recherche des économies d'échelle et de variété. Il fallait ensuite tirer les conséquences de tout cela et dans le rapport salarial et dans le rapport d'approvisionnement.

$\mathrm{Au}$ premier niveau, il convenait d'affaiblir ou de casser les statuts spécifiques sous lesquels évoluaient les différentes catégories de personnel des industries de défense. Une nouvelle fois, l'objectif était de "banalisent" leurs pratiques professionnelles et d'introduire des possibilités d'ajuster plus rapidement et librement les effectifs et les compétences aux besoins des managers, sans se priver d'aller chercher ces compétences dans des entreprises d'autres secteurs.

$\mathrm{Au}$ second niveau, le rapport d'achat marqué par les exigences technologiques et organisationnelles très spécifiques du secteur semblait lui aussi devoir être radicalement révisé pour que les nouveaux industriels puissent via de vrais marchés accéder au meilleurs prix aux inputs requis par leurs fabrications et bénéficier, dans leur entreprise de réduction des coûts, des avantages de la dualité. Vu de l'amont, pour les sous-traitants de la défense, la même évolution impliquait un "changement de culture" qui ressortait comme le prix à payer pour voir leurs débouchés pérennisés et éventuellement diversifiés. Pour les entreprises, qui, jusqu'alors, ne travaillaient pas pour la défense, s'ouvrait alors potentiellement un domaine d'opportunités nouveau.

En invoquant un "exemple anglais" présenté plus haut, on a donc, dans les années 90, assisté à l'émergence d'un véritable projet de désinstitutionnalisation-réinstitutionnalisation de l'industrie qui

159 Economie et Institutions $-\mathrm{n}^{\circ} 12 \& 13-2008-2009$ 
s'est traduit par de lourdes évolutions règlementaires et institutionnelles. Il a d'abord été porté par les Etats désireux d'encaisser les "dividendes de la paix". Dans la mesure où il impliquait aussi un mouvement de privatisation et une ouverture à la concurrence, il a été assez largement soutenu par les investisseurs et les grandes entreprises privées gravitant autour de ces activités. La presse d'affaire et une bonne partie des économistes l'ont soutenu et une partie des derniers a mis certains de ses outils et en particulier ceux que fournit la théorie des incitations à son service. A l'inverse, sans toujours, s'exprimer explicitement contre cette entreprise politique, une très large part des acteurs de l'industrie et, au premier chef, les armées ont œuvré pour que soit préservé l'essentiel de l'ancien système. Comme l'a montré S. Moura (2007), quelles qu'aient pu être les évolutions du périmètre et du contrôle des entreprises du secteur et celles des procédures d'achat mises en œuvre par "l'Etat Client", le rapport commercial a au fond peu évolué et le "lien symbiotique" s'est perpétué limitant le rôle de la concurrence aux marges du système. Le même travail a montré que bien qu'apparemment soumis dans la même période aux impératifs de la "financiarisation", les industriels de la défense y ont in fine trouvé essentiellement un moyen de pression supplémentaire sur leurs Etats pour obtenir d'eux les moyens de développer leurs matériels et de servir correctement leurs actionnaires.

C'est au fond sur les rapports les moins centraux pour l'ordre institutionnel caractéristique des industries de défense que sont le rapport salarial et le rapport d'achat que l'entreprise aura eu le plus d'effets "banalisant" (Moura, 2008). L'affaiblissement des statuts et surtout les redéfinitions de périmètre des entreprises qui ont accompagné l'activité très forte que l'on a enregistrée sur le marché du contrôle a impliqué un ajustement assez rapide des effectifs. Surtout, les grandes entreprises ont changé, elles, leurs pratiques d'achat et fait peser sur leurs fournisseurs une pression à la baisse des coûts très fortes sans toutefois relâcher leurs exigences en matière de qualité, de secret et d'excellence technologique. Elles ont ainsi orchestré chez leurs sous-traitants un mouvement darwinien de regroupement et de sélection très perceptible dans toutes les grandes régions productrices d'armes et, en particulier, dans celles sur lesquelles nous nous sommes penchés et qui se sont effectivement trouvées avec l'ensemble de ces évolutions confrontées à des problèmes dits de reconversion.

En utilisant le prisme que nous proposons ici, les dits problèmes apparaissent sous l'angle de toute leur diversité et l'on peut alors saisir leurs différences de traitement par les différents SLAP.

Dans la mesure où les dividendes de la paix se sont d'abord traduits par des réductions d'effectifs associées parfois à des

160 Economie et Institutions - n²12\&13-2008-2009 
fermetures de sites, la reconversion peut d'abord concerner les personnels qu'il s'agit alors de "requalifier" pour qu'ils puissent réinvestir localement leurs compétences dans d'autres rapports salariaux. La spécificité de leurs compétences, liées à celles des technologies et des exigences associees aux productions d'armes d'une part et à celles des cultures professionnelles d'autre part, rend l'entreprise difficile. Plus spécifiquement, dans des régions périphériques dont la spécialisation est relativement forte précisément parce qu'on avait décidé de les y implanter ou de les y développer en raison des insuffisances de leur industrialisation, les secteurs d'accueil sont rares ou inexistants. Cela constitue une forte incitation à ne pas lâcher la proie pour l'ombre et à privilégier dans l'action publique la préservation de ce qui peut l'être contre d'hypothétiques diversifications des entreprises ou du tissu. De ce point de vue, le marché de l'emploi de la Lombardie s'oppose assez clairement à ceux du North-West anglais ou d'Aquitaine. Ces différences expliquent en partie qu'on ait pu privilégier un temps en Lombardie l'option radicale alors que re-spécialisation et ambigüité ont été les maîtres mots dans les deux autres régions.

Si le rapport salarial a représenté une contrainte et un enjeu majeur des politiques locales d'aide aux reconversions, le cœur des dispositifs d'aide déployées en direction des grands établissements et, surtout, des entreprises sous-traitantes a plutôt visé le rapport commercial et surtout le rapport d'approvisionnement.

S'agissant du rapport commercial, on a effectivement assisté dans certains cas à des efforts pour en modifier la teneur et faire en sorte que les grands établissements travaillant pour l'ASD développent des applications civiles des technologies dont ils avaient acquis la maîtrise en travaillant à concevoir et à produire des armes. Ainsi, en Aquitaine, dans le domaine des matériaux composites hautes performances pour lequel le pôle missilier et avions de combat avait acquis une certaine avance, on a imaginé que des matériels sportifs puissent être développés et offrir aux industriels et à leurs sous-traitants des débouchés alternatifs. Etant donnés les différentiels de coûts acceptables et, surtout, le déficit de culture commerciale et marketing qu'impliquait le travail pour les armées dans le cadre de relations exclusives principalement centrées sur la performance technologique, les équipes dédiés à ces projets sont demeurées en position marginale et ces tentatives ont tourné court. Même au sein de groupes diversifiés dans l'aéronautique et le spatial, militaire et civil, il ressort que les établissements dédiés aux deux types de marchés sont distincts et relèvent de divisions distinctes entre lesquelles les synergies sont assez limitées. Les velléités des SLAP d'en favoriser le développement pèsent peu par rapport à l'organisation des groupes et à ces choix de spécialiser leurs sites. Dans ce contexte, contrairement aux espoirs initialement mis sur la

161 Economie et Institutions $-\mathrm{n}^{\circ} 12 \& 13-2008-2009$ 
dualité et le potentiel de diversification qu'elle devait offrir, le choix de reconfiguration du rapport commercial apparait, au fil des ans, plutôt binaire : rester dans l'ASD et renoncer à valoriser ailleurs ses compétences technologiques ou renoncer à l'ASD pour se donner les moyens d'être à même d'apprendre à travailler autrement. La trajectoire suivie par le SLAP anglais renvoie de fait au premier des termes de l'alternative, le radicalisme lombard des débuts au second. Pour ce qui est de l'Aquitaine, on peut penser que les responsables des dispositifs d'aide partageraient assez volontiers l'analyse mais qu'ils se sont gardés de le mettre en avant pour favoriser selon les cas, et avec les mêmes dispositifs, l'une ou l'autre option stratégique.

Enfin, s'agissant du cœur de cible des politiques publiques d'aides aux reconversions - qui la plupart du temps n'en sont pas -, elles visent le rapport d'approvisionnement et renvoient fondamentalement aux mêmes problématiques. Du reste, ce que nous désignons comme rapport d'achat des fabricants d'armes est, pour leurs fournisseurs, un rapport commercial. Dans la mesure où il est structuré comme une relation de sous-traitance très exclusive, le rapport en question fait bien peu intervenir les compétences commerciales et en limite le développement. Dans la mesure où diversifier son activité impliquerait de la part des entreprises concernées qu'elles apprennent à intégrer leur activité dans les rapports d'approvisionnement d'autres activités, on va trouver là une limite majeure pour l'action publique. Cette limite est d'autant plus forte que dans la période visée la pression à la baisse des coûts dans l'ASD a exigé des entreprises concernées de lourds efforts de rationalisation et d'investissement dédiés aux industries de défense. On aurait pu imaginer que la dite pression aurait rendu la soustraitance de l'ASD moins " exotique " et aurait facilité la vente de ses services à d'autres clients. Il ne semble pas que, en règle générale, cela ait été le cas car les actifs acquis alors ressortent comme assez difficilement redéployables car ils renvoient à des cahiers des charges que l'on ne retrouve pas dans la plupart des autres activités. Ainsi relativement à leur passé, les entreprises qui ont continué de travailler pour l'ASD ont effectivement satisfait la requête de leur donneur d'ordres. Dans la mesure toutefois où il ne s'est en aucun cas agi de "rogner sur la qualité ", les efforts consentis n'ont pas permis de dualiser l'activité. Parce qu'elles ne parvenaient plus à suivre ou parce qu'elles avaient pris conscience de ce que continuer de travailler pour l'ASD ne pouvait que les enfermer dans cette spécialisation, certaines entreprises ont effectivement renoncé et ont pu bénéficier pour cela, en Aquitaine en particulier, du soutien du SLAP.

Ce troisième éclairage de nos trois cas permet ainsi de saisir que la banalisation des industries de défense a, pour l'essentiel,

162 Economie et Institutions - nº $12 \& 13-2008-2009$ 
correspondu à une rationalisation / sélection de la filière qui s'est faite sous l'égide du couple Etats/grandes entreprises de défense. Via la financiarisation et les privatisations, ceci a déclenché des regroupements d'importance qui ont permis de renouveler le cœur du système en même temps que la pression à la baisse des coûts sans relâchement de l'exigence technologique conduisait à une respécialisation de la sous-traitance. Ce processus a été géré sur des bases essentiellement nationales qui ont laissé les autres niveaux de régulation en position marginale : aux niveaux européen et régional, on a alors eu à accompagner un mouvement sur lequel on n'avait pas prise.

\section{Conclusion}

Dans les marges toutefois, les régulateurs européens et locaux ne sont pas en position homologues. En effet, alors que les premiers doivent se contenter des généralités et de faire des chèques, les seconds sont à la tête d'administrations expertes et ont en face d'eux les traductions concrètes des réorganisations qui sont décidées aux méta-niveaux. Ils en mesurent le sens et ajustent mois après mois leur diagnostic sur ce qu'il est possible ou non de faire. Ceci les conduit de fait dans la seconde moitié des années 90 à saisir que si des groupes duaux existent bien, des établissements ou des PME diversifiés sont en revanche tout à fait improbables. Dès lors, ils peuvent avec ce temps d'avance qu'ils ont sur l'UE soit renoncer à ce type de dispositifs (cas lombard) soit les utiliser clairement au service d'un renforcement de l'ASD renouvelée (cas anglais) soit sérier les cas de re-spécialisation et de conversion pour utiliser les mêmes aides dans l'une ou l'autre perspective (cas aquitain). Dans la mesure où la relation d'achat est fondamentale et est fortement localisée, l'aide des SLAP aux grands établissements afin de soutenir leur SPT parait relativement efficace lorsque la coordination est assurée par des opérateurs qui sont au cœur des systèmes nationaux : c'est ce qui se passe dans les cas anglais -où BAe domine l'architecture locale - et aquitain -où c'est le DRIRD, fonctionnaire du Ministère de la défense, qui domine. On peut alors se demander s'il n'y a pas là une forme de détournement des fonds européens censément dédiés à l'objectif de reconversion externe. En effet lorsqu'il s'agit au contraire de conversion, la réussite est plus problématique car les chances de succès sont moindres et que l'expertise des SLAP est également moins avérée.

Ceci signifie que ces politiques publiques régionales méritent d'être conçues et évaluer des trois points de vue que nous avons adoptés ici successivement. Les systèmes sectoriels, les systèmes productifs territorialisés et les systèmes locaux d'action publique se renvoient nécessairement les uns aux autres et ne sont tout 
simplement pas pensables les uns sans les autres. Ceci résulte en partie de ce que les "structures industrielles" sont moins déterminées par les technologies, comme le prétendent les analyses standards, qu'elles ne le sont par la structure politique et institutionnelle des régulations qui s'appliquent aux différents niveaux pertinents. Dès lors les chances de succès des politiques régionales méritent d'être jaugées à l'aune de ces structures puisque c'est une forme de capacité des SLAP à refléter ces structures pour les diffracter qui leur confèrent leurs capacités à maximiser leurs effets aux marges.

S'agissant du niveau d'intervention européen, il apparaît dans notre cas bien peu opérant dès lors qu'aucun des RI n'est principalement régulé à ce niveau d'une part et que comme régulateur sans administration l'UE semble particulièrement peu à même d'acquérir la maîtrise des processus sur lesquels elle entend intervenir.

Si la première des caractéristiques est assez spécifique du secteur, la seconde l'est moins et révèle un premier niveau d'incomplétude des dispositifs européen d'intervention sur les industries : parce que sa capacité à faire exécuter ses décisions ou à en contrôler les effets est très limitée l'UE est obligée de s'en remette aux Etats qui peuvent produire des dispositifs européens des interprétations extrêmement variables, à l'image de ce que nous constations pour les SLAP utilisant les fonds Konver. Ainsi même lorsque c'est l'UE qui dit la loi - comme ce peut être le cas en matière de concurrence -, les paysages - et les tribunaux - nationaux peuvent être le théâtre d'interprétations de "l'intention du législateur " très hétérogènes (Jullien, 2008).

S'agissant pour terminer de la marginalité de l'intervention européenne dans la structuration des RI, on la retrouve à un degré moindre dans la plupart des industries. En effet, si souvent, l'un au moins des RI est régulé à Bruxelles, ce n'est presque jamais le cas de tous les dispositifs en même temps. Dès lors, l'européanisation des industries est presque fatalement un processus incomplet puisque les complémentarités institutionnelles nécessaires ont, presque toujours, à être configurées nationalement (Jullien, Smith, 2008)

164 Economie et Institutions - n¹2\&13-2008-2009 


\section{Bibliographie}

Barnier F. (1996), La civilisation des armes, Thèse de doctorat sociologie, Université Paris VII, Presse Universitaire du Septentrion, Villeneuve d'Ascq.

Beckouche P. (1996), La nouvelle géographie de l'industrie aéronautique européenne, L'Harmattan, Paris.

CESA (Conseil Économique et Social d'Aquitaine) (1992), Les Perspectives des industries de l'aéronautique, de l'espace et de la défense en Aquitaine, Rapport présenté par J. Bonneville, Bordeaux, 15 Juin, Multig.

Chesnais F. et C. Serfati (1992), L'armement en France, Nathan, Paris.

CRCI-A (Chambre Régionale de Commerce et d'Industrie d'Aquitaine) (1994), Situation des entreprises de sous-traitance du secteur aéronautique spatial et militaire en Aquitaine, Bordeaux, Juin, Multig.

DDA (Defence Diversification Agency) (2000), "Defence Diversification: Getting the most out of defence technology", White Paper - Final Text.

Economic Geography (1993), "Special Issue: Defense Spending and regional Development", 69 (2).

Fligstein N. (2001), The Architecture of Markets. An Economic Sociology of Twenty-First Century Capitalist Societies, Princeton University Press, Princeton.

Frigant V. et B. Jullien (1997), "La reconversion des industries de la défense en Gironde", Communication présentée au XXXIIİ̀me colloque de l'ASRDLF, Lille, 1-3 septembre.

Frigant V. et S. Moura (2004), "Les déterminants des stratégies réactives des sous-traitants de la défense. Le cas des PME Aquitaines, Lombardes et du North-West dans la décennie 1990", Revue Internationale PME, 17 (3/4), pp.121-145.

Graziola G. et S. Parazzini (2001), "The Defence Industry in Lombardy during the 1990s : Adjustment and Repositioning", Communication à la conférence internationale, Disarmament, Arms Industries and Conversion in the Regions, Bremen, 27-28 april.

Grossetti M. et alii (1994), Evaluation des Fonds de Restructuration de la Défense (FRED), Rapport pour la Direction Aux Restructurations (DAR) du Ministère de la Défense, CERS, Toulouse, Multig.

GSAD (Gruppo di Studio su Armi e Disarmo) (1996), L'industria militare lombarda negli anni 90: tedenze, propettive $e$ ruolo della politica economica, Rapporto Finale per l'Agenzia Regionale per la riconversione dell'industria bellica, 3 volumes, Milano. 
Hébert J-P. (1995), Production d'armement, mutation du système français, Notes et Etudes Documentaires $\mathrm{n}^{\circ} 5009, \mathrm{La}$ documentation Française, Paris.

Hébert J-P. (2001), "La consolidation de l'Europe de l'armement face au défi transatlantique", Cahier d'Etudes Stratégiques, $\mathrm{n}^{\circ} 30$, CIRPES, EHESS, Paris.

Jalabert G. (1974), Les industries aéronautiques et spatiales en France, Privat, Toulouse.

Jullien B. (1995), De la diffusion à la dilution d'une technologie: le cas des matériaux composites. Pour une approche de la continuité des processus de changements technoéconomiques, Thèse de doctorat ès sciences économiques, Université Bordeaux I, Multig.

Jullien B. (2008), "European Automobile Distribution: Globalization and Incomplete Liberalization", in Jullien B. et A. Smith (eds), Industries and Globalization: The Political Causality of Difference, Palgrave, London, pp.28-57.

Jullien B. et A. Smith (2008), 'L'Union européenne et la régulation des industries : vers une sociologie politique de l'économie", Politique européenne, 25, pp.137-159.

Jullien B. et A. Smith (eds) (2008), Industries and Globalization: The Political Causality of Difference, Palgrave, London.

Kirat T. et D. Bayon (2006), Les marchés publics de la défense, Bruylant, Bruxelles.

Lazonick W. et A. Prencipe (2005), "Dynamic Capabilities and Sustained Innovation: Strategic Control and Financial Commitment at Rolls-Royce plc", Industrial and Corporate Change, 14 (3), pp.501-542.

LCC (Lancashire County Council) (1994), Restructuring of the Defence Industry: The Regional View Lancashire, March, Preston, Multig., 15p.

Ministry of Defence (MoD) (1998), Defence White Paper: A http://www.mod.uk/issues/diversification/

diversification_wp.htm

Moura S. (2007), L'impossible banalisation des industries de défense, Thèse de Doctorat es sciences économiques, GREThA, Université Montesquieu-Bordeaux IV, 24 Septembre, Bordeaux, Multig.

Moura S. (2008), “The US Defence Industry since 1945 : Globalization Refused", in Jullien B. et A. Smith (eds), Industries and Globalization: The Political Causality of Difference, Palgrave, London, pp.129-148.

Nelson R. et S. Winter (1977), "Dynamic Competition and Technical Progress", in Balassa B. et R. Nelson (eds.), Economic 
Progress, Private Values, and Public Policy: Essays in Honor of William Fellner, North-Holland, Amsterdam.

Perani G. (2000), "Italian Contrasts in Regional Military Industrial Conversion", International Regional Science Review, 23 (1), pp. 91-102.

Sachwald F. (1999), Banalisation et restructuration des industries de défense, Les Notes de l'IFRI $n^{\circ} 15$, Série Transatlantique.

Versailles D., Mérindol V. et P. Cardot (2003), La recherche et la Technologie, enjeux de puissance, Economica, Paris.

167 Economie et Institutions $-\mathrm{n}^{\circ} 12 \& 13-2008-2009$ 